\title{
A importância da Educação Alimentar e Nutricional como estratégia para idosos hipertensos: Uma revisão integrativa
}

The importance of Food and Nutrition Education as a strategy for hypertensive elderly: An integrative review

La importancia de la Educación en Alimentación y Nutrición como estrategia para ancianos hipertensos: Una revisión integrativa

Sâmia Camille Correia Lima

ORCID: https://orcid.org/0000-0002-2735-738X Faculdade do Vale do Jaguaribe, Brasil E-mail: samiadcorreia19@gmail.com

Cristiane Souto Almeida

ORCID: https://orcid.org/0000-0002-1571-2452 Faculdade do Vale do Jaguaribe, Brasil E-mail: cristiane.souto@fvj.br

Livia Viviane Guimarães do Couto

ORCID: https://orcid.org/0000-0001-7249-7229 Faculdade do Vale do Jaguaribe, Brasil E-mail: liviacoutonutri@gmail.com Jamile de Lima Santos

ORCID: https://orcid.org/0000-0001-9456-9895 Escola de Saúde Pública do Ceará, Brasil E-mail: jamillelimajbl@gmail.com

Marcília Ingrid Lima Barroso Nunes

ORCID: https://orcid.org/0000-0002-5871-3930 Universidade do Estado do Rio Grande do Norte, Brasil E-mail: prof.marciliabarroso@gmail.com

Silvana Medeiros de Araújo

ORCID: https://orcid.org/0000-0003-1296-7422

Universidade do Estado do Rio Grande do Norte, Brasil

E-mail: silvanamedeiros87@hotmail.com

Paloma Katlheen Moura Melo

ORCID: https://orcid.org/0000-0002-9265-038X

Universidade do Estado do Rio Grande do Norte, Brasil E-mail: palomakatlheen@hotmail.com

\begin{abstract}
Resumo
A educação alimentar e nutricional é uma importante ferramenta que tem como objetivo primordial proporcionar a todas as pessoas que as utilizam o bem-estar físico, além de prevenir o aparecimento ou agravamento de patologias, tornando possível assim maior longevidade com o máximo de qualidade de vida. O presente estudo trata-se de uma revisão integrativa elaborada metodologicamente segundo o manual de Diretrizes Metodológicas do Ministério da Saúde e auxílio do Preferred Reporting Items for Systematic Reviews and Meta-Analyses Protocols. O processo de pesquisa foi realizado nas bases de dados The Scientific Electronic Library Online, Biblioteca Virtual em Saúde e PubMed (National Library of Medicine), com a utilização no período correspondente aos meses de janeiro a junho de 2021. Os resultados encontrados por meio da pesquisa eletrônica nas bases de dados totalizaram 8 artigos. Quanto as discussões, a mesma têm como objetivo facilitar o entendimento sobre a importância da educação alimentar e nutricional dos idosos. Conclui-se que a Educação Alimentar e Nutricional, poderá atuar de forma ativa no processo de envelhecimento humano e pode ser considerada uma estratégia para promover uma alimentação adequada e saudável, e dessa forma promover saúde e qualidade de vida, trabalhando com ferramentas interdisciplinares e ativas voltadas a pessoa idosa no contexto da Hipertensão Arterial Sistêmica.
\end{abstract}

Palavras-chave: Hipertensão; Idosos; Educação alimentar e nutricional.

\section{Abstract}

Food and nutrition education is an important tool whose primary objective is to provide all people who use them with physical well-being, in addition to preventing the onset or worsening of pathologies, thus making possible greater longevity with maximum quality of life. The present study is an integrative review prepared methodologically 
according to the Manual of Methodological Guidelines of the Ministry of Health and the assistance of Preferred Reporting Items for Systematic Reviews and Meta-Analyses Protocols. The search process was carried out in The Scientific Electronic Library Online, Virtual Health Library and PubMed (National Library of Medicine) databases, with use in the period corresponding to the months of January to June 2021. The results found through the electronic search in the databases totaled 8 articles. As for the discussions, the aim is to facilitate the understanding of the importance of food and nutrition education for the elderly. It is concluded that Food and Nutrition Education can act actively in the human aging process and can be considered a strategy to promote adequate and healthy eating, and thus promote health and quality of life, working with interdisciplinary and active tools aimed at the elderly in the context of Systemic Arterial Hypertension.

Keywords: Hypertension; Seniors; Food and nutrition education.

\section{Resumen}

La Educación alimentaria y Nutricional es una herramienta importante cuyo objetivo primordial es brindar bienestar físico a todas las personas que las utilizan, además de prevenir la aparición o agravamiento de patologías, posibilitando así una mayor longevidad con la máxima calidad de vida. El presente estudio es una revisión integradora elaborada metodológicamente de acuerdo con el Manual de Lineamientos Metodológicos del Ministerio de Salud y la asistencia de Ítems Preferidos de Reporte para Revisiones Sistemáticas y Protocolos de Metanálisis. El proceso de búsqueda se realizó en las bases de datos The Scientific Electronic Library Online, Virtual Health Library y PubMed (National Library of Medicine), con uso en el período correspondiente a los meses de enero a junio de 2021 . Los resultados encontrados a través de la búsqueda electrónica en el las bases de datos totalizaron 8 artículos. En cuanto a las discusiones, el objetivo es facilitar la comprensión de la importancia de la educación alimentaria y nutricional para las personas mayores. Se concluye que la Educación Alimentaria y Nutricional puede actuar de forma activa en el proceso de envejecimiento humano y puede ser considerada una estrategia para promover una alimentación adecuada y saludable, y así promover la salud y la calidad de vida, trabajando con herramientas interdisciplinarias y activas dirigidas a las personas mayores en el contexto de la hipertensión arterial sistémica.

Palabras clave: Hipertensión; Mayores; Educación alimentaria y nutricional.

\section{Introdução}

O envelhecimento é um ciclo natural de todo o ser humano, e apresenta as seguintes características: “[...] é universal, por ser natural, não depende da vontade do indivíduo, todo ser nasce, desenvolve-se, cresce, envelhece e morre. É irreversível, apesar de todo o avanço da medicina [...] nada impede o inevitável fenômeno, nem o faz reverter" (Duarte, 2008).

Segundo a Organização Mundial da Saúde (OMS) é considerado idoso nos países em desenvolvimento, os indivíduos com 60 anos ou mais (Santos; Gomes \& Lima; 2018). Tendo a presença de alterações particulares do ciclo de envelhecimento que tornam o sujeito mais tendencioso para desenvolver por exemplo a hipertensão arterial, sendo a mesma a principal doença crônica nessa faixa etária.

Segundo Ferreira et al., (2012) uma promoção do envelhecimento saudável se mostra complexa, pois gira em torno não apenas da qualidade de vida, mas também do acesso a serviços de saúde que auxiliem o idoso neste processo.

A Educação Alimentar e Nutricional (EAN) é conceituada como um componente de atuação multiprofissional, que garante o conhecimento e aprendizado permanentes, que propõe os educandos a desenvolver a autonomia e a voluntariedade diante dos hábitos alimentares saudáveis, com a utilização de recursos e abordagens educativas ativas e problematizadoras (Brasil, 2012b).

Dessa forma, a Educação Alimentar e Nutricional faz parte de uma importante linha na para promover de hábitos alimentares saudáveis, sendo uma tática indispensável que está compreendida nas políticas públicas em alimentação e nutrição (Boog, 2013).

É possível compreender então como a Educação Alimentar e Nutricional torna-se importante no que se refere às políticas públicas de alimentação e nutrição por conta da necessidade de aumentar a discussão sobre as possibilidades, limitações e o jeito como a Educação Alimentar e Nutricional é atingida, procedendo em ações governamentais, especialmente as que são desenvolvidas pelos Ministérios do Desenvolvimento Social e de Combate à Fome, levando então para a construção do Marco 
de Referência de Educação Alimentar e Nutricional das Políticas Públicas, que faz parte de uma importante etapa de valorização dessas atuações (Greenwood \& Fonseca, 2016).

Na Atenção Primária a Saúde o desenvolvimento das ações parte de métodos educativos que possam interferir no processo de saúde-doença da população. Portanto, a alimentação e a nutrição fazem parte das condições básicas para a promoção e proteção à saúde, sendo que as estratégias de EAN representam papel fundamental no contexto da Atenção Básica e, em especial, na Estratégia Saúde da Família (ESF), que apareceu para realinhar a organização da atenção com o intuito de aproximar os trabalhos educativos nas comunidades, expandindo o seu campo de intervenções (Alencar et al., 2016; Brasil, 2012a; Jaime et al., 2011; Oliveira \& Pereira, 2013).

Assim, a Hipertensão Arterial é considerada atualmente uma doença de alta prevalência no Brasil e no mundo, sendo um dos principais complicadores para as doenças cardiovasculares, que são as primeiras causas de mortalidade em todo mundo (Nascente et al., 2010).

Para Cervato et al., (2005) o processo ativo que envolve transformações no modo de pensar, sentir e agir dos indivíduos e pelo qual eles adquirem, mudam ou reforçam conhecimentos, atitudes e práticas conducentes à saúde é definida como uma educação nutricional. Ferramenta esta, que dá autonomia, para que a pessoa possa assumir, com plena consciência, a responsabilidade por seus atos relacionados à alimentação.

As práticas educativas devem ter como base o diálogo, e, em se tratando da saúde dos idosos, esta abordagem deve ser feita com maior apreço e cuidado para que se crie um vínculo educador-educando, promovendo a autoestima do idoso e devolvendo-lhe o sentido de humanidade (Assis, 2002). Considerando o potencial da alimentação em promover melhores condições de saúde elegeu-se a Educação Alimentar e Nutricional como estratégia a fim de contribuir com a qualidade de vida dos idosos. (Hardt et al., 2006),

Assim, o objetivo deste estudo é apresentar evidências científicas disponíveis na literatura sobre as estratégias de educação alimentar e nutricional na saúde do idoso com HAS.

\section{Metodologia}

O presente estudo trata-se de uma revisão integrativa elaborada metodologicamente segundo o manual de Diretrizes Metodológicas do Ministério da Saúde e auxílio do Preferred Reporting Items for Systematic Reviews and Meta-Analyses Protocols (PRISMA).

Refere-se a revisão integrativa da literatura, a qual identifica, seleciona, coleta dados, analisa e avalia criticamente estudos sobre um determinado assunto, a partir de uma pergunta norteadora, que obedece a métodos sistemáticos e explícitos (Lima et al., 2016).

\subsection{Estratégia De Busca}

O processo de pesquisa foi realizado nas bases de dados SCIELO (The Scientific Electronic Library Online), BVS (Biblioteca Virtual em Saúde) e PubMed (National Library of Medicine), com a utilização de palavras-chave em Português e Inglês no período correspondente aos meses de janeiro a junho de 2021.

A escolha de palavras-chave foi realizada baseado no PICOS (P- população; I- intervenção; C- comparador; ODesfecho; S- tipo de estudo), sendo a população correspondente a idosos com idade igual a 60 anos ou mais com hipertensão, a intervenção sendo Educação alimentar e nutricional direcionada ao hipertenso, o comparador sendo grupo controle, o desfecho sendo Redução da Pressão Arterial Sistêmica do idoso através da Educação Alimentar e Nutricional e os estudos do tipo observacionais e experimentais através da pesquisa transversal. 
As palavras-chaves que formaram a tática de busca foram "Hipertensão", "Idosos" e "Educação Alimentar e Nutricional" em português, sendo traduzida simultaneamente para o inglês "Hypertension", "Seniors" e "food and Nutrition education". Sendo determinadas através dos Descritores de Ciências da Saúde (DeCS), no Portal da Biblioteca Virtual em Saúde (BVS) e do Medical Subject Headings (MeSH).

Em conjunto as palavras-chaves formaram os termos de busca unidos pelos operadores booleanos (AND e OR): “(Food and Nutrition education) OR (nutritional intervention) AND (Seniors) AND (Hypertension), em inglês (Educação alimentar e nutricional) OR (intervenção nutricional) AND (idosos) AND (hipertensão) em português.

\subsection{Critérios de Inclusão e Exclusão}

Os critérios de inclusão foram: (1) homens e mulheres com hipertensão arterial sistêmica; (2) idosos com idade $\geq$ a 60 anos; (3) artigos científicos ou periódicos publicados do ano de 2010 a maio de 2021; (4) disponibilizados na íntegra; (5) artigos em inglês e português; (6) estudos abordando a educação alimentar e nutricional e qualidade de vida para idosos hipertensos.

Os critérios de exclusão foram: (1) artigos que abordassem Educação Alimentar e Nutricional, mas não relacionavam a HAS em idosos; (2) artigos publicados que tratem de doenças crônicas não transmissíveis de maneira geral; (3) artigos pagos; (4) artigos ou estudos que façam associação da Hipertensão Arterial Sistêmica com outra patologia ou não mencionem a Educação Alimentar e Nutricional.

\subsection{Seleção Dos Estudos}

Nessa etapa de revisão, foram exportados os artigos das bases de dados selecionadas (The Scientific Electronic Library Online) e BVS (Biblioteca Virtual em Saúde) para o software EndNote X9๑, no qual em seguida foi feita a remoção de duplicatas. Que por fim, migrou-se para o Microsoft WORD 2016 ®, para a seleção dos títulos, resumos e textos completos.

$\mathrm{Na}$ etapa seguinte, foi feito a leitura dos títulos, desses títulos selecionados os resumos e por fim, os artigos lidos na íntegra para a aplicação dos critérios de inclusão e exclusão no estudo.

\subsection{Avaliação Metodológica}

Para a avaliação metodológica do estudo, foi usado o instrumento AMSTAR (Assessment of Multiple Systematic Reviews), que foi elaborado com base na análise e na atualização de outros instrumentos. Ele tem como função avaliar as revisões de cunho sistemático de ensaios randomizados. A classificação geral de confiança nos resultados de pesquisas da revisão fica dividida em alta, moderada, baixa e criticamente baixa e quanto aos domínios que serão avaliados, eles se dividem em 16 criteriosos pontos que foram julgados como importantes para a qualidade metodológica da revisão (Shea et al., 2009; Shea et al., 2017).

Em síntese, é possível afirmar que é uma ferramenta prática para pesquisas feitas por profissionais de saúde que não possuem treinamento avançado em epidemiologia. Através dela é possível realizar uma avaliação de forma rápida e capaz de ser reproduzida no quesito de qualidade de revisões sistemáticas, com estudos randomizados ou não com intervenções em saúde, ou os dois (Shea et al., 2009; Shea et al., 2017).

\section{Resultados}

Após seguir todos os critérios de buscas o número de artigos encontrados foi de 736 artigos, sendo 537 resultados no Scielo, 87 resultados Portal da Biblioteca Virtual em Saúde: BVS (BIREME); e 112 resultados do PubMed. 
Desses 736 artigos, foram tirados 332 estudos por estarem com títulos duplicados, restando 404 artigos para leitura de título. Após isso 383 artigos foram excluídos a partir da leitura dos resumos, reduzindo assim em 21 artigos para leitura na íntegra, dos quais 13 foram excluídos por tratarem apenas de hipertensão arterial sistêmica, sem mencionar Educação Alimentar e Nutricional e abordarem a hipertensão em adultos com idade menor a 60 anos. Por fim, 8 artigos foram inclusos na revisão, como demonstrado na Figura 1.

Os oito artigos escolhidos, seguiram todos os critérios de inclusão, abordando Hipertensão Arterial Sistêmica e Educação Alimentar e Nutricional em adultos com idade maior ou igual a 60 anos.

Desses estudos foram extraídos os seguintes dados: autores, ano, local, amostra, objetivo, principais resultados obtidos e conclusão.

Após os artigos passarem por esta seleção, foram reunidos de acordo com a tabela 2, contendo o autor, ano, local, amostra, objetivo, principais resultados e conclusão. 
Figura 1 - Fluxograma de processo de seleção dos estudos.
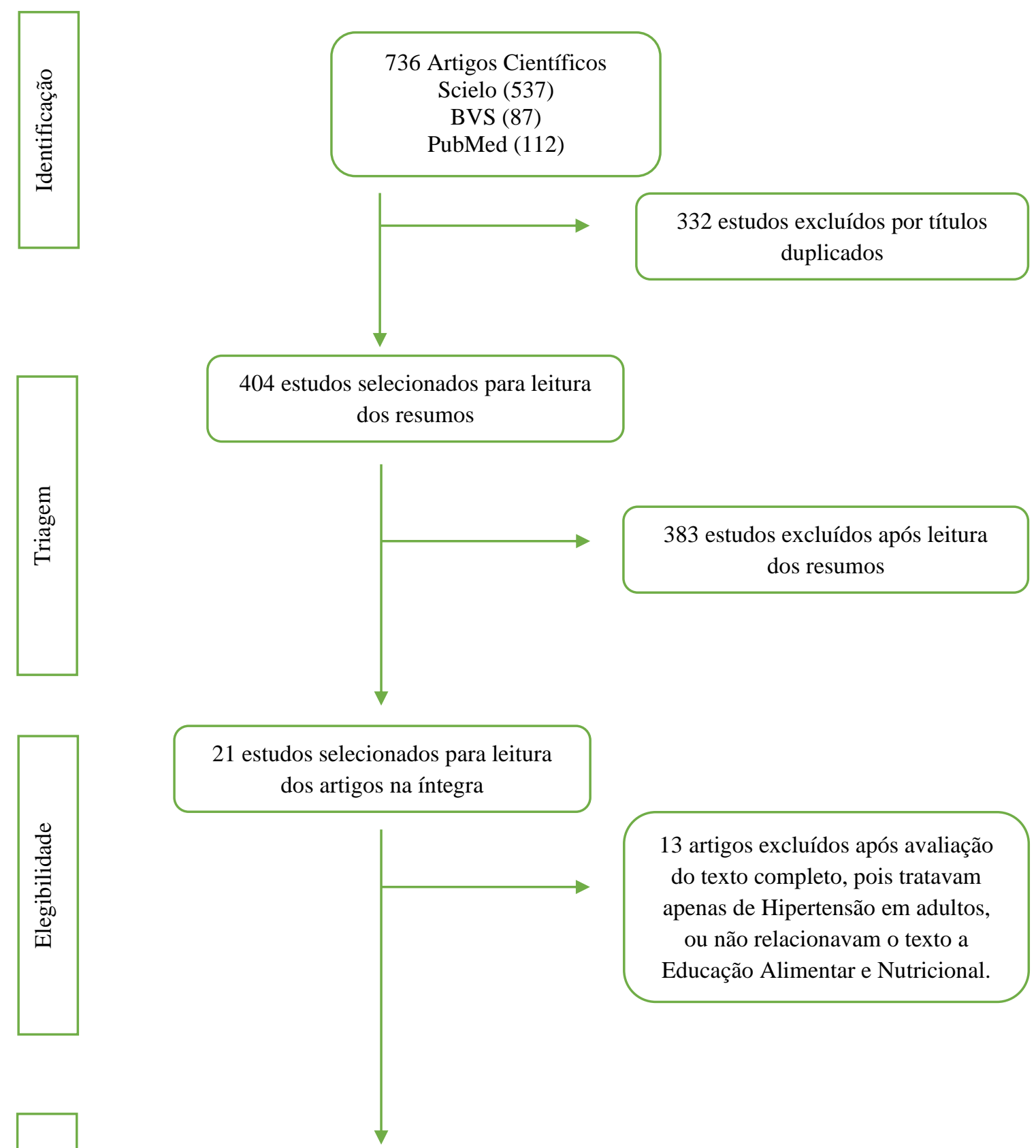

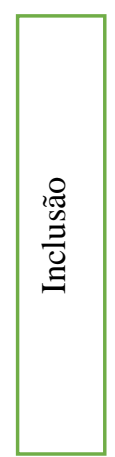

8 artigos incluídos na revisão integrativa

Fonte: Dados da Pesquisa (2021). 


\subsection{Características Dos Estudos}

Os artigos inclusos são estudos de características qualitativas ou quantitativas, desenvolvidos a partir de estudos de caso e revisões sistemáticas da literatura, e contando ao todo com 599 participantes, do sexo feminino e masculino, com idade a partir de 60 anos. E fizeram ao longo do seu desenvolvimento correlação entre a Educação Alimentar e Nutricional, Hipertensão Arterial Sistêmica, Controle da HAS através da Educação Alimentar e Nutricional buscando assim melhorar a qualidade de vida de idosos.

O primeiro estudo foi publicado em 2008, no Brasil, e não relatou qualquer tipo de financiamento. Teve a participação inicial de 19 de sujeitos, com idade entre 61 e 79 anos, dos quais 2 eram do sexo masculino e 17 do sexo feminino. No que tange às motivações apresentadas pelos idosos, quanto à sua participação na oficina oferecida pelo Programa Terceira Idade em Ação/UFPI, os entrevistados se posicionaram segundo três abordagens: na primeira, eles descortinaram o processo de gerontologia educacional, via Universidades da Terceira Idade; na segunda, emergiram novas perspectivas para uma velhice ativa, participativa e segura em articulação com os aspectos biopsicossociais do ser humano; e na terceira, refletiram sobre questões do âmbito das políticas públicas de saúde e nutrição (Alencar, Junior \& Carvalho, 2008).

O segundo estudo, foi publicado em 2009, em São Paulo, e não relatou qualquer tipo de financiamento. O trabalho foi pautado na metodologia do Estudo de Caso da pesquisa qualitativa em saúde com os seus principais instrumentos: entrevistas gravadas e encontros em grupo. Ocorreram nove encontros para discussão sobre alimentação e nutrição com um grupo de nove idosos, na Universidade Aberta à Terceira Idade, do Município de Araraquara/SP, no período de agosto a outubro de 2007 (Criscuolo, 2009).

Os resultados do estudo indicaram que todos os participantes tentaram se apropriar dos conhecimentos, bem como consolidar e aprofundar conhecimentos anteriores, que foram discutidos nos encontros em grupo, através do processo de reflexão e conscientização, trazendo benefícios de forma individual e coletiva. $\mathrm{O}$ trabalho de educação nutricional associado à educação problematizadora evidenciou contribuições para a construção de conhecimentos dos indivíduos, às mudanças comportamentais e para o convívio social, favorecendo na prevenção e na promoção da saúde, sendo, portanto, uma alternativa para o planejamento das políticas públicas relacionadas à saúde dos idosos (Criscuolo, 2009).

O terceiro estudo foi publicado no ano de 2014, realizado também no Brasil, não relatou financiamento. Participaram do estudo 150 idosos, destes, 66,7\% eram mulheres; a idade dos participantes variou de 60 a 92 anos, com média de 69,9 anos (dp $\pm 7,5$ ). As variáveis com diferenças significativas entre homens e mulheres foram: estado conjugal, renda, escolaridade e aposentadoria, sendo maior o percentual de homens casados, com renda de 2SM ou mais, com escolaridade de cinco anos de estudo ou mais e que recebiam aposentadoria. Em geral, houve predomínio em ambos os sexos de idosos casados, de cor de pele branca, com três ou mais filhos vivos e baixa escolaridade (White \& Léon, 2014).

Embora sem diferença estatisticamente significativa, os homens referiram maior frequência de consulta com nutricionista que as mulheres, tanto nos últimos três meses (40\% e 28\%) quanto nos últimos 12 meses (68\% e 32\%), e alguma vez na vida (90\% e $74 \%)$. No estudo, $80 \%$ e $64 \%$ dos homens e das mulheres, respectivamente, afirmaram ter recebido algum tipo de material educativo por escrito sobre orientações nutricionais. É alta a frequência de dúvidas em relação às orientações (62\% dos homens e 50\% das mulheres). E ainda, $49 \%$ das mulheres e 50\% dos homens relataram falta de vontade para comer o que foi recomendado, e $49 \%$ das mulheres e $42 \%$ dos homens não comem nos horários recomendados (White \& Léon, 2014).

Em relação às orientações nutricionais, todos os idosos relataram recebimento de orientações relacionadas à necessidade de consumo de verduras, frutas e água; no entanto, as orientações não eram seguidas por uma parcela dos entrevistados. Somente $28,7 \%$ consumiam verduras diariamente, e a grande maioria $(91,4 \%)$ consumia apenas uma porção por refeição (dados não apresentados). Quanto às frutas, 73,3\% consumiam diariamente, porém apenas uma porção. Somente 13,3\% bebiam menos de cinco copos de água por dia (White \& Léon, 2014). 
Em relação ao consumo de leite desnatado, 93,3\% receberam orientação e 74,3\% da amostra relataram consumo diário, mas apenas de um copo/dia. Embora quase $40 \%$ não tenham recebido orientação sobre a necessidade de consumo de leguminosas, os percentuais em todas as categorias eram muito semelhantes aos que receberam orientação, atingindo quase 90\% nas categorias de maior consumo. Apenas em relação aos alimentos integrais foi observado consumo significativamente maior nos idosos que receberam a orientação (12,1\% e 2\% de consumo diário) (White \& Léon, 2014).

A parcela que não cumpria com as orientações de consumo diário variou segundo o alimento: alimentos integrais $(87,9 \%)$, leguminosas $(78,3 \%)$, verduras $(71,3 \%)$, frutas $(26,7 \%)$ e leite desnatado ou semidesnatado $(25,7 \%)$. Todos os idosos referiram ter recebido orientação para diminuir o consumo de sal, mas somente 33,3\% relataram restringi-lo. Já a orientação para restringir o consumo de tempero pronto foi recebida por $41,3 \%$ dos indivíduos, e quanto ao cumprimento desta restrição não houve diferença em relação aos que não receberam orientação (59\% e 58\%). A restrição do consumo de alimentos ricos em gordura animal atingiu 92,7\%, mas seu consumo semanal foi alto (63,6\% nos que não receberam orientação e 59\% nos que foram orientados) (White \& Léon, 2014).

Assim, a porcentagem de não cumprimento das restrições foi de 66,7\% para sal, $41 \%$ para temperos industriais e $69 \%$ para gordura animal. A respeito da importância do horário das refeições, $88,7 \%$ declararam ter recebido esta orientação, sendo que apenas $34,6 \%$ a seguem adequadamente; $52,6 \%$ ficam mais de cinco horas sem comer e 12,8\% comem o tempo todo portanto 65,4\% não cumprem a recomendação de horário das refeições (tabela 3). Quanto às orientações sobre a manutenção ou perda de peso, $82 \%$ receberam esta orientação, mas o percentual que ganhou peso foi um pouco superior nos que foram orientados (26\% e 22,2\%) (White \& Léon, 2014).

O quarto estudo foi realizado no ano de 2015, e assim como os demais foi produzido no Brasil e não relatou financiamento. Dos 41 idosos avaliados, 20 eram do sexo masculino (48,8\%) e 21 do sexo feminino $(51,2 \%)$. A idade média encontrada foi de 73,17 anos, com idade mínima de 61 anos e máxima de 99 anos, DP $\pm 8,91$. Houve predominância da faixa etária entre 60 e 69 anos (41,5\%), seguida da faixa etária de 70 a 79 anos (39\%) e 80 anos e mais (19,5\%). Quanto à raça, $73,2 \%$ são brancos, $24,2 \%$ negros e $2,2 \%$ pardos. Do total de idosos, $58,5 \%$ apresentaram o ensino fundamental incompleto, 9,8\% o ensino fundamental completo e um número considerável de idosos eram analfabetos, representando 19,5\%. A estatura média encontrada foi de 1,60 m, mínima de 1,40 m e máxima de 1,68 m (Henriques et al., 2015).

A média do peso corporal encontrada foi $65,1 \mathrm{~kg}$, com peso mínimo de 35,6 kg e o máximo de 95,0 kg. Em relação à classificação do Índice de Massa Corporal, 27\% dos idosos apresentaram baixo peso, 27\% eutrofia e 46\% sobrepeso. As mulheres idosas apresentaram maior frequência de IMC $\geq 27$ (76,1\%), o que foi classificada como sobrepeso, ao passo que os idosos do sexo masculino apresentaram IMC $\geq 27$ (20\%). Por outro lado, eles apresentaram IMC $<22$ (classificado como baixo peso) em maior frequência (40,0\%) em relação às mulheres idosas $(9,5 \%)$. O sexo apresentou associação significativa em relação à classificação do estado nutricional ( $\mathrm{p}=0,004)$ (Henriques et al., 2015).

A medida da circunferência da cintura, por meio da qual se traçou o risco de doenças cardiovasculares, apresentou uma média de $91,43 \mathrm{~cm}, \mathrm{DP} \pm 15,14$, sendo a mínima de $58 \mathrm{~cm}$ e a máxima de $119 \mathrm{~cm}$. Em relação ao risco de complicações metabólicas substancialmente aumentadas, as mulheres apresentaram percentual mais elevado $(76,0 \%)$ em relação aos homens $(15,0 \%)$, considerando que, para o sexo feminino, a medida da circunferência para risco aumentado é de $\geq 88$ centímetros e do masculino é de $\geq 102$ centímetros. Foram verificadas medidas de pressão arterial sistólica (P.A.S.) e diastólica (P.A.D.). Os resultados obtidos apresentaram uma média de P.A.S de 126,34 mmhg, sendo a mínima de $90 \mathrm{mmhg}$ e a máxima de 180 mmhg. A P.A.D. mostrou uma média de 80,49 mmhg (Henriques et al., 2015).

Em relação aos resultados obtidos com a aferição da pressão arterial, foi possível afirmar que $29 \%$ apresentaram níveis pressóricos normais, seguidos de 27\% com hipertensão arterial, 22\% com níveis considerados ótimos e 12\% com níveis limítrofes $1 \%$ e $10 \%$ correspondentes aos estágios 2 e 3, respectivamente. Quanto às principais doenças de prevalência nos 
idosos foram relatadas 46 moléstias, com predominância da hipertensão arterial $(32,61 \%)$, seguida da diabetes mellitus $(21,74 \%)$. Entre os entrevistados, 28,2\% relataram não apresentar doenças. Em relação à quantidade de medicamentos, a média de uso foi de 2,2 medicamentos por pessoa idosa, com prevalência de uso dos anti-hipertensivos e hipoglicemiantes, resultado já esperado considerando as citadas comorbidades que mais apareceram entre os entrevistados (Henriques et al., 2015).

A aderência ao tratamento medicamentoso também foi um item analisado. Entre os entrevistados, $37 \%$ apresentaram alta adesão, 29\% média adesão e os $34 \%$ restantes apresentaram baixa adesão ou não utilizavam medicamentos. Sobre os marcadores alimentares, pode-se afirmar que grande parte dos entrevistados consome alimentos considerados saudáveis pelo menos seis dias por semana, como por exemplo, 44,4\% consomem salada crua, 32,4\% legumes e verduras frescas cozidas, $54,8 \%$ frutas frescas ou salada de frutas, $87,5 \%$ feijão e $64,4 \%$ leite ou iogurte. Dos alimentos considerados não saudáveis os resultados mostram que 36,7\% consomem bolachas/biscoitos salgados ou salgadinhos de pacote; $27,3 \%$, bolacha/biscoito recheados, doces ou chocolates; e 20\%, refrigerantes pelo menos seis vezes na semana. Um percentual significativo relata não consumir ou consumir um dia na semana os seguintes alimentos: frituras $86,6 \%$, hambúrguer e embutidos $60 \%$, refrigerantes $30 \%$, bolachas/biscoitos salgados ou salgadinhos de pacote 33,3\% (Henriques et al., 2015).

Com base no levantamento do perfil alimentar e nutricional de pessoas idosas foram identificados seis principais problemas dessa população, tais como analfabetismo, distúrbios nutricionais (baixo peso e sobrepeso), risco de complicações metabólicas substancialmente aumentadas, presença de doenças crônicas não transmissíveis (HA e DM), baixa adesão ao tratamento medicamentoso e consumo alimentar inadequado. Diante disso, foi proposto um plano de ação para o acompanhamento e o monitoramento das condições alimentares e nutricionais dos idosos. Para cada situação problema foram descritos os possíveis responsáveis para sua execução, o período, o local onde seria realizado, o porquê de adotar tal medida, como fazer e o custo (Henriques et al., 2015).

O quinto estudo foi publicado em 2016, no Rio Grande do Norte, e não relatou qualquer tipo de financiamento. Tratava-se de uma revisão sistemática de literatura, de publicações voltadas à saúde e qualidade de vida da pessoa idosa. Foi elaborado pelo método dedutivo, apontado por Severino (2007), não continha amostra e apresentou como objetivo principal exibir os benefícios de praticar atividade física com enfoque na prevenção de doenças, e para melhorar a qualidade de vida da pessoa idosa (Lima \& Pietsak, 2016).

O estudo mostrou que a prática de atividade física, gerou benefícios para a saúde da pessoa idosa, trazendo melhor qualidade de vida, e prevenindo algumas doenças, como exemplo, as doenças crônicas não transmissíveis. Foi visto também, que o idoso que possui uma vida fisicamente ativa, tem uma melhor interação com a família e amigos, o que, consequentemente, pode estar associado com o melhor desempenho mental do idoso (Lima \& Pietsak, 2016).

O sexto estudo foi publicado em 2017, no Rio de Janeiro, o trabalho teve como objetivo revisar de forma sistemática as produções científicas acerca de intervenções da educação alimentar e nutricional com sujeitos adultos na Atenção Primária à Saúde no Brasil, no período de 2006 a 2016. Foram selecionados 11 artigos que responderam aos critérios de elegibilidade: ser artigos originais, de acesso livre, ser publicado nos periódicos de 2006 a 2016, propor intervenções e/ou avaliações de atividades envolvendo a educação nutricional na APS, participadores com idade maior que 20 anos, independente do sexo, nível socioeconômico e etnia, e artigos desenvolvidos no Brasil (França \& Carvalho, 2017).

O estudo destacou como principais consequências das práticas educativas desenvolvidas que elas possuem pouca abordagem em relação a promoção da saúde e uma forte tendência metodológica clássica, fazendo necessário mais estudos qualitativos, na percepção da autonomia, e que expandam o uso de metodologias ativas nas ações de intervenção (França \& Carvalho, 2017).

O sétimo estudo foi publicado em 2017, no Pará e não relatou qualquer tipo de financiamento. Tratava-se de um estudo feito a partir do modelo transversal, aleatório, de características descritivas e analíticas, realizado no período de junho 
de 2015 a setembro de 2016. Com o objetivo de descrever o perfil socioeconômico, antropométrico, dietético e identificar a prevalência de doenças crônicas em idosos. Sua amostra contava com 185 idosos com predomínio do sexo feminino na faixa etária de 60 a 69 anos (Miranda et al., 2017).

Foi aplicado um questionário semiestruturado para obtenção das seguintes variáveis: identificação (sexo, data do nascimento etc.), estilo de vida (ingestão de bebida alcoólica, hábito de fumar, prática de atividade física) e a apresentação ou não de doenças crônicas não degenerativas, dados que costumam influenciar questões relacionadas à saúde. Na antropometria, foi mensurado peso (Kg) e altura (m) para obtenção do índice de massa corporal (IMC), dobra cutânea tricipital (PCT), circunferência do braço (CB), circunferência muscular do braço (CMB) e circunferência da panturrilha (CP) (Miranda et al., 2017).

As análises estatísticas dos dados foram realizadas com o auxílio dos softwares Microsoft Office Excel e o programa BioEstat 5.0 (AYRES et al., 2007). As estimativas das prevalências foram apresentadas em proporções (\%), com seus respectivos intervalos de confiança de 95\% (IC95\%). A análise de variância (ANOVA) e a comparação de Tukey foram utilizadas para verificar o efeito do grupo etário nas variáveis antropométricas, quando a amostra apresentava normalidade, e o teste de Kruskal-Wallys para dados com distribuição não normal. O nível de significância foi fixado em $p<0,05$ (Miranda et al., 2017).

O principal resultado foi de que se detectou em sua maioria a presença acentuada de sedentarismo e de um estado nutricional de sobrepeso/obesidade, segundo o índice de massa corpórea; as outras medidas compartimentadas apresentaram eutrofia, prevalência de hipertensão arterial e diabetes mellitus e grande ingestão de carboidratos simples e baixo consumo de alimentos protetores (Miranda et al., 2017).

Este último estudo foi realizado no ano de 2020, e inicialmente foi realizado o cadastro dos idosos hipertensos da Estratégia Saúde da Família Lagoa do município de Paulistana (PI), através de fichas contendo as seguintes informações: dados pessoais, socioeconômicos e hábitos alimentares, além da medicação usada para a hipertensão (com as devidas dosagens e horários de administração) que foram anexadas aos seus prontuários (Martins \& Fé, 2020).

O acompanhamento do projeto de intervenção foi realizado mensalmente durante as reuniões na sede da UBS através da aplicação de questionários para conhecimento dos hábitos alimentares dos pacientes pela nutricionista do NASF I e o uso de planilhas para a anotação da pressão arterial dos idosos, que foram aferidas por uma enfermeira ou técnica de enfermagem da ESF (Martins \& Fé, 2020).

Após ser avaliada a aceitabilidade do sal de ervas entre os idosos hipertensos, foi sugerido a realização de um grupo operativo para discutir e debater a opinião deles sobre a apreciação de ervas como tempero de alimentos. A partir das respostas dadas, a nutricionista do NASF I elaborou um relatório resumindo o grau de aceitabilidade entre os participantes e a sua relação com o controle dos seus níveis pressóricos (Martins \& Fé, 2020).

Os resultados obtidos durante os encontros do projeto foram apresentados mensalmente em reuniões aos gestores, coordenação da Atenção Básica e profissionais da saúde do município. Tais resultados foram divulgados através de relatórios com gráficos elaborados pela nutricionista do NASF I, mostrando a evolução dos participantes do projeto quanto ao controle dos seus níveis pressóricos e melhoria dos seus hábitos alimentares (Martins \& Fé, 2020).

Foi realizada a aplicação de questionários aos usuários após o término do projeto para avaliação do conhecimento adquirido com a intervenção, além de suas sugestões e críticas (Martins \& Fé, 2020).

As principais características de cada estudo, distribuição dos artigos de acordo com autores, ano, local, amostra, objetivo, resultados e conclusão inclusos nesse artigo científico estão (Tabela 1). 
Tabela 1: Distribuição dos artigos segundo autores, ano, local, amostra, objetivo, principais resultados e conclusão.

\begin{tabular}{|c|c|c|c|c|c|c|}
\hline AUTOR & ANO & LOCAL & AMOSTRA & OBJETIVO & PRINCIPAIS RESULTADOS & CONCLUSÃO \\
\hline $\begin{array}{l}\text { Alencar, } \\
\text { Barros } \\
\text { Carvalho }\end{array}$ & 2008 & Piauí & $\begin{array}{l}\text { A amostra do estudo } \\
\text { constituiu-se de } 19 \text { de } \\
\text { sujeitos, com idade entre } \\
61 \text { e } 79 \text { anos, dos quais } 2 \\
\text { eram do sexo masculino e } \\
17 \text { do sexo feminino. }\end{array}$ & $\begin{array}{l}\text { Analisar a contribuição da } \\
\text { educação nutricional a partir } \\
\text { das suas dimensões } \\
\text { sociopolíticas que permeiam a } \\
\text { promoção da saúde. }\end{array}$ & $\begin{array}{l}\text { Os depoimentos dos idosos revelaram, como } \\
\text { aspectos motivacionais para ingressar no } \\
\text { programa e cursar a oficina, o ensino- } \\
\text { aprendizagem, a integração social e o cuidado } \\
\text { com a saúde. As concepções sobre a contribuição } \\
\text { da educação nutricional para a promoção da } \\
\text { saúde foram categorizadas nas temáticas: } \\
\text { capacidade funcional e autocuidado. }\end{array}$ & $\begin{array}{l}\text { Nesse contexto, foi possível contemplar } \\
\text { como os idosos estão vivenciando os seus } \\
\text { processos de envelhecimento e quais } \\
\text { mudanças, no campo da promoção da } \\
\text { saúde, o Programa Terceira Idade em } \\
\text { Ação/UFPI, tem propiciado quanto ao seu } \\
\text { bem-estar físico, mental e social. }\end{array}$ \\
\hline Criscuolo & 2009 & São Paulo & 160 idosos & $\begin{array}{l}\text { Buscar evidências das } \\
\text { contribuições da educação } \\
\text { nutricional associada à } \\
\text { educação problematizadora de } \\
\text { Paulo Freire junto a um grupo } \\
\text { de idosos. }\end{array}$ & $\begin{array}{l}\text { Os resultados indicaram que todos os } \\
\text { participantes tentaram se apropriar dos } \\
\text { conhecimentos, bem como consolidar e } \\
\text { aprofundar conhecimentos anteriores, que foram } \\
\text { discutidos nos encontros em grupo, através do } \\
\text { processo de reflexão e conscientização, trazendo } \\
\text { benefícios de forma individual e coletiva. }\end{array}$ & $\begin{array}{l}\text { O trabalho de educação nutricional } \\
\text { associado à educação problematizadora } \\
\text { evidenciou contribuições para a } \\
\text { construção de conhecimentos dos } \\
\text { indivíduos, àn às mudanças } \\
\text { comportamentais e para o convívio } \\
\text { social, favorecendo na prevenção e na } \\
\text { promoção da saúde, sendo, portanto, } \\
\text { uma alternativa para o planejamento das } \\
\text { políticas públicas relacionadas à saúde } \\
\text { dos idosos. }\end{array}$ \\
\hline $\begin{array}{l}\text { White \& } \\
\text { León }\end{array}$ & 2014 & São Paulo & $\begin{array}{l}\text { Participaram } 150 \text { idosos, } \\
\text { sendo } 66,7 \% \text { do sexo } \\
\text { feminino, com } \\
\text { média de idade de } 69,9 \\
\text { anos }(\mathrm{dp} \pm 7,5) \text {. }\end{array}$ & $\begin{array}{l}\text { Descrever aspectos do } \\
\text { atendimento nutricional e das } \\
\text { práticas alimentares entre } \\
\text { idosos em face das } \\
\text { orientações } \quad \text { nutricionais } \\
\text { recebidas. }\end{array}$ & $\begin{array}{l}\text { Os homens, mais do que as mulheres, estiveram } \\
\text { em acompanhamento nutricional regular nos } \\
\text { últimos } 12 \text { meses ( } 68 \% \text { e } 32 \%) \text {. Apesar de a } \\
\text { grande maioria dos entrevistados relatar } \\
\text { recebimento de orientações nutricionais, o } \\
\text { consumo diário recomendado não era atendido } \\
\text { para alimentos integrais ( } 88 \%) \text {, verduras ( } 71 \%) \text {, } \\
\text { frutas ( } 27 \%) \text {; não restringiam sal (67\%) e gordura } \\
\text { animal (65\%) e ainda } 65 \% \text { não cumpriam horário } \\
\text { de refeições. Disseram ter recebido material } \\
\text { educativo contendo orientações nutricionais: } 80 \% \\
\text { dos homens e } 64 \% \text { das mulheres. No entanto, } \\
50 \% \text { das mulheres e } 62 \% \text { dos homens relataram }\end{array}$ & $\begin{array}{l}\text { A baixa adoção das recomendações } \\
\text { nutricionais aponta a necessidade de } \\
\text { melhorar a capacitação dos profissionais } \\
\text { de saúde, para que possam orientar de } \\
\text { forma eficiente os pacientes quanto ao } \\
\text { consumo alimentar. Esta constatação } \\
\text { reforça a importância da atuação de uma } \\
\text { equipe de saúde multiprofissional que } \\
\text { motive os idosos a aderirem ao } \\
\text { tratamento, viabilizando melhor controle } \\
\text { das doenças crônicas e prevenção de } \\
\text { complicações secundárias. }\end{array}$ \\
\hline
\end{tabular}




\begin{tabular}{|c|c|c|c|c|c|}
\hline $\begin{array}{l}\text { Henriques et } \\
\text { al., }\end{array}$ & 2015 & $\begin{array}{l}\text { Minas } \\
\text { Gerais }\end{array}$ & 41 idosos & $\begin{array}{l}\text { Apresentar o perfil alimentar } \\
\text { e nutricional de pessoas } \\
\text { idosas, visando monitorá-las } \\
\text { por meio de plano de ação } \\
\text { estratégico. }\end{array}$ & $\begin{array}{l}\text { Os resultados mostraram que houve } \\
\text { predominância do sexo feminino }(51,2 \%) \text {, média } \\
\text { de idade de } 72,6 \text { anos, primeiro grau incompleto } \\
(58,5 \%) \text { e risco de complicações metabólicas } \\
\text { entre as idosas ( } 76 \%) \text {; na classificação do Índice } \\
\text { de Massa Corporal (IMC), } 27 \% \text { apresentaram } \\
\text { baixo peso e } 46 \% \text {, sobrepeso. }\end{array}$ \\
\hline
\end{tabular}

$\begin{array}{lll}\text { Lima \& } 2016 & \text { Apresentar os benefícios da } \\ \text { Pietsak } & \text { prática de atividade física } \\ & \text { para a prevenção de doenças, } \\ & \text { e para melhorar a qualidade } \\ & \text { de vida da pessoa idosa. }\end{array}$

França \&

2017

Rio de

11 artigos

Carvalho

Janeiro

\section{Foi}

Foi visto, através de alguns estudos realizados, que a vida inativa propicia a aceleração dos sintomas do processo de envelhecimento, assim como o surgimento de patologias relacionadas à idade, e, em contrapartida, o estudo mostrou que os idosos que possuem uma vida fisicamente ativa, possuem uma melhor qualidade de vida, quando se trata da saúde, e de interações com amigos e familiares.
Concluiu-se que o processo de envelhecimento resulta em diferentes fatores de risco e enfermidades, incluindo as alimentares e nutricionais que, uma vez identificados e diagnosticados, necessitam de acompanhamento e de monitoramento pelos gestores e pelas equipes de saúde.

O estudo mostrou que a prática de atividade física, gera benefícios para a saúde da pessoa idosa, trazendo melhor qualidade de vida, e prevenindo algumas doenças, como exemplo, as doenças crônicas. Foi visto também, que o idoso que possui uma vida fisicamente ativa, tem uma melhor interação com a família e amigos, o que, consequentemente, pode estar associado com o melhor desempenho mental do idoso.

Faz-se necessária uma deliberação política para desenvolver ações de EAN dentro da APS, com descrição das atividades no planejamento dos municípios e das práticas educativas nas unidades, que possibilitem o investimento e o apoio da gerência e logística necessária para o seu efetivo desenvolvimento a fim de garantir não só a realização, mas também a continuidade dessas atividades dentro dos serviços de saúde. 


\begin{tabular}{|c|c|c|c|c|c|c|}
\hline $\begin{array}{l}\text { Miranda et } \\
\text { al., }\end{array}$ & 2017 & Pará & $\begin{array}{l}185 \text { idosos } \text { com } \\
\text { predomínio do sexo } \\
\text { feminino na faixa } \\
\text { de } 60 \text { a } 69 \text { anos. }\end{array}$ & $\begin{array}{l}\text { Descrever o perfil } \\
\text { socioeconômico, } \\
\text { antropométrico, dietético e } \\
\text { identificar a prevalência de } \\
\text { doenças crônicas em idosos. }\end{array}$ & $\begin{array}{l}\text { Detectou-se em sua maioria a presença acentuada } \\
\text { de sedentarismo e de um estado nutricional de } \\
\text { sobrepeso/obesidade, segundo o índice de massa } \\
\text { corpórea; as outras medidas compartimentadas } \\
\text { apresentaram eutrofia, prevalência de hipertensão } \\
\text { arterial e diabetes mellitus e grande ingestão de } \\
\text { carboidratos simples e baixo consumo de } \\
\text { alimentos protetores. }\end{array}$ & $\begin{array}{l}\text { A presença acentuada de sedentarismo, } \\
\text { estado nutricional de sobrepeso/obesidade } \\
\text { em sua maioria, prevalência de } \\
\text { hipertensão arterial e diabetes mellitus, } \\
\text { grandes ingestões de carboidratos simples } \\
\text { e baixo consumo de alimentos protetores } \\
\text { foram identificados neste estudo. Assim, } \\
\text { essas informações ressaltam a a } \\
\text { importância da educação nutricional } \\
\text { atrelada a uma vigilância alimentar, com } \\
\text { o intuito de evitar o aumento dos riscos } \\
\text { relacionados a presença } \\
\text { desenvolvimento de doenças crônicas. }\end{array}$ \\
\hline Martins \& Fé & 2020 & Piauí & - & $\begin{array}{l}\text { Orientar os idosos hipertensos } \\
\text { da Unidade Básica de Saúde } \\
\text { Lagoa do município de } \\
\text { Paulistana (PI) sobre a } \\
\text { importância da adoção de } \\
\text { hábitos alimentares saudáveis } \\
\text { como tratamento não } \\
\text { medicamentoso para controle } \\
\text { da hipertensão. }\end{array}$ & $\begin{array}{l}\text { Foram realizadas orientações sobre alimentação } \\
\text { saudável utilizando diferentes técnicas } \\
\text { educativas, acompanhamento dos pacientes } \\
\text { através de consultas nutricionais individualizadas } \\
\text { e o desenvolvimento das oficinas "Sal de Ervas". }\end{array}$ & $\begin{array}{l}\text { Esperou-se que com a implantação deste } \\
\text { projeto ocorra à estabilização das cifras } \\
\text { de pressão arterial, e a população idosa } \\
\text { hipertensa fique mais informada sobre } \\
\text { alimentação saudável, consciente dos } \\
\text { alimentos que não pode ingerir, logrando } \\
\text { a diminuição das complicações causadas } \\
\text { pela hipertensão, além do melhor } \\
\text { acompanhamento deste grupo de } \\
\text { pacientes. }\end{array}$ \\
\hline
\end{tabular}




\subsubsection{Educação Alimentar e Nutricional no envelhecimento e fator de controle da pressão arterial.}

A educação nutricional no envelhecimento apresenta-se como uma atitude inovadora aos novos desafios e às demandas alimentares e nutricionais geradas pela emergência de um novo segmento etário e de uma nova fase do curso de vida: a velhice (Alencar, Junior \& Carvalho, 2008).

A implementação da Educação Alimentar visa a redução de fatores de risco e de danos evitáveis da pessoa idosa, com base em um elenco de estratégias interdisciplinares e integradas por diferentes pontos de atenção à saúde, que resultarão na melhoria da qualidade de vida das populações. A elaboração do plano de ações irá contribuir para o acompanhamento e o monitoramento da pessoa idosa nas áreas associadas das unidades saúde da família por meio de ações estratégicas intersetoriais (Henriques et al., 2015).

Desta forma, a educação nutricional, dentro do âmbito das políticas públicas, cumpre o seu objetivo de fornecer subsídios e agir preventivamente para que os indivíduos sejam capazes de preservar a sua autonomia e suas funções físicas e mentais, contribuindo para uma velhice saudável e influenciando o impacto das doenças nos serviços de saúde a longo prazo (Criscuolo, 2009).

Entre as pessoas idosas, a hipertensão arterial é um fator determinante de morbidade e mortalidade, mas quando adequadamente controlada, reduz significativamente as limitações funcionais e modificam a história natural (Martins \& Fé, 2020). Com isso, a educação nutricional que é um processo que engloba mudanças de atitudes, maneira de pensar, agir e sentir, sempre estimulando e reforçando os conhecimentos sobre saúde, permitindo assim, que o idoso se mantenha longe do risco de desnutrição ou de outras doenças ligadas ao consumo em excesso de alimentos (Cervato et al.,2005).

A intervenção da Educação Alimentar e Nutricional tem como objetivos gerais a prevenção primária e secundária da HAS e como objetivos específicos: redução dos níveis pressóricos, possibilitando a diminuição da quantidade de fármacos utilizados na terapia medicamentosa, manutenção do peso corporal, redução da obesidade visceral e redução de peso, nos casos de sobrepeso e obesidade; adequação do consumo energético e de macro e micronutrientes, conforme necessidades individuais; valorização dos hábitos e da cultura alimentar, assim como de uma alimentação saudável, promovendo ações de reeducação alimentar (Brasil, 2013).

\subsubsection{Avaliação da Qualidade Metodológica}

A presente revisão foi submetida a avaliação metodológica utilizando a ferramenta Assessment of Multiple Systematic Reviews (AMSTAR), com aplicação de checklist (Quadro 1), verificando todos os aspectos metodológicos para construção do presente trabalho, possuindo classificação de alta qualidade por não possuir falhas críticas, apresentando os resultados dos estudos de forma precisa e abrangente (Shea et al., 2017).

A avaliação metodológica feita a partir da ferramenta Assessment of Multiple Systematic Reviews (AMSTAR), com aplicação do checklist (Quadro 1) foi classificada como revisão de alta qualidade. 
Quadro 1. Avaliação da qualidade metodológica da revisão (Checklist AMSTAR).

\begin{tabular}{|c|c|}
\hline \multicolumn{2}{|l|}{ Revisão de alta qualidade } \\
\hline $\begin{array}{l}\text { 1. As perguntas da pesquisa e os critérios de inclusão para a revisão incluíram os componentes do } \\
\text { PICO? }\end{array}$ & Sim \\
\hline $\begin{array}{l}\text { 2. O relatório da revisão continha uma declaração explícita de que os métodos de revisão foram } \\
\text { estabelecidos antes da realização da revisão e justificou algum desvio significativo do protocolo? }\end{array}$ & Parcial Sim \\
\hline 3. Os autores da revisão explicaram sua seleção dos desenhos do estudo para inclusão na revisão? & Sim \\
\hline 4. Os autores da revisão usaram uma estratégia abrangente de busca na literatura? & Sim \\
\hline 5. Os autores da revisão realizaram a seleção do estudo em duplicado? & Sim \\
\hline 6. Os autores da revisão executaram a extração de dados em duplicado? & Sim \\
\hline 7. Os autores da revisão forneceram uma lista de estudos excluídos e justificaram as exclusões? & Sim \\
\hline 8. Os autores da revisão descreveram os estudos incluídos em detalhes adequados? & Sim \\
\hline $\begin{array}{l}\text { 9. Os autores da revisão usaram uma técnica satisfatória para avaliar o risco de viés (RoB) em } \\
\text { estudos individuais que foram incluídos na revisão? RCT }\end{array}$ & Sim \\
\hline NRSI & $\mathbf{0}$ \\
\hline $\begin{array}{l}\text { 10. Os autores da revisão relataram as fontes de financiamento para os estudos incluídos na } \\
\text { revisão? }\end{array}$ & Sim \\
\hline $\begin{array}{l}\text { 11. Se a metanálise foi realizada, os autores da revisão utilizaram métodos apropriados para a } \\
\text { combinação estatística de resultados? RCT }\end{array}$ & - \\
\hline NRSI & - \\
\hline $\begin{array}{l}\text { 12. Se a metanálise foi realizada, os autores da revisão avaliaram o impacto potencial do RoB em } \\
\text { estudos individuais sobre os resultados da metanálise ou outra síntese de evidências? }\end{array}$ & - \\
\hline $\begin{array}{l}\text { 13. Os autores da revisão foram responsáveis pelo RoB em estudos individuais ao interpretar / } \\
\text { discutir os resultados da revisão? }\end{array}$ & Sim \\
\hline $\begin{array}{l}\text { 14. Os autores da revisão forneceram uma explicação satisfatória para e discussão de alguma } \\
\text { heterogeneidade observada nos resultados da revisão? }\end{array}$ & Sim \\
\hline $\begin{array}{l}\text { 15. Se eles realizaram síntese quantitativa, os autores da revisão realizaram uma investigação } \\
\text { adequada do viés de publicação (viés de pequeno estudo) e discutiram seu provável impacto nos } \\
\text { resultados da revisão? }\end{array}$ & Sim \\
\hline $\begin{array}{l}\text { 16. Os autores da revisão relataram alguma fonte potencial de conflito de interesses, incluindo } \\
\text { algum financiamento recebido pela realização da revisão? }\end{array}$ & Não \\
\hline
\end{tabular}

Fonte: Dados da Pesquisa (2021).

\section{Discussão}

O processo natural do envelhecimento provoca modificações na vida da pessoa idosa, e essas modificações podem ainda ser agravadas pela presença de diversas patologias, além disso, a nutrição e a alimentação na terceira idade ainda são pouco exploradas, não tendo recebido a devida atenção (Lima \& Pietsak, 2016).

A procura aumentada dos idosos por atendimentos de saúde, principalmente entre portadores de doenças crônicas, e a necessidade de prevenir as complicações descrevendo aspectos do atendimento nutricional e das práticas alimentares entre idosos em face das orientações nutricionais recebidas (White \& León, 2014).

A presença de doenças nutricionais, como baixo peso e sobrepeso, bem como das doenças crônicas, mostram o grande desafio dos profissionais de saúde na adesão farmacoterapêutica e na adoção de consumo alimentar adequado por parte das pessoas idosas (Henriques et al., 2015). É, portanto, nesse enfoque que a Educação Alimentar Nutricional se destaca entre os distintos estudos epidemiológicos, nos quais os resultados mostraram uma conexão entre comportamento alimentar e as alterações do processo saúde-doença. (Alencar, Barros Júnior \& Carvalho, 2008).

A preocupação com o estado nutricional e a alimentação dessa população está ligada a vários fatores relacionados ao 
Research, Society and Development, v. 10, n. 9, e8010917832, 2021

(CC BY 4.0) | ISSN 2525-3409 | DOI: http://dx.doi.org/10.33448/rsd-v10i9.17832

envelhecimento, uma vez que as mudanças fisiológicas, sociais e psicológicas pelas quais a pessoa passa no decorrer da vida geram mudanças no padrão de alimentação e nutrição, podendo levar à perda excessiva de peso, à falta de apetite e à diminuição do estímulo alimentar (Henriques et al., 2015).

Entre os hábitos de vida, a alimentação ocupa um papel de destaque no tratamento e prevenção da HAS. Várias modificações dietéticas demonstram benefícios sobre a pressão arterial, como a redução da ingestão de sal e álcool, redução do peso e possivelmente aumento no consumo de alguns micronutrientes, como potássio e cálcio (Martins \& Fé, 2020).

Sendo assim, é possível alcançar, por meio da promoção da saúde e prevenção de doenças, um envelhecimento saudável, preservando a capacidade funcional do idoso pelo maior tempo possível (Alencar, Barros Júnior \& Carvalho, 2008).

A Educação Alimentar e Nutricional (EAN) pode ser considerada uma estratégia para promover uma alimentação adequada e saudável, e dessa forma promover saúde e qualidade de vida, garantindo o Direito Humano a uma Alimentação Adequada (DHAA), assegurando assim a Segurança Alimentar e Nutricional (SAN) (Brasil, 2013).

Sendo um processo multifatorial, a educação alimentar e nutricional caracteriza-se por alterações do padrão alimentar e de composição corporal dos indivíduos a partir de mudanças sociais, econômicas, demográficas, tecnológicas e culturais que afetam diretamente o estilo de vida e o perfil de saúde da população. As principais mudanças consistem na adoção de um padrão dietético com alto teor de gorduras saturadas e açúcares e com baixo teor de fibras. Tal padrão alimentar somado à redução dos níveis de atividade física resulta em crescimento das taxas de excesso de peso entre os brasileiros (Santos et al., 2013).

Sendo assim, a Educação Alimentar e Nutricional trabalha na promoção da qualidade de vida de idosos para que eles possam usufruir de um envelhecimento ativo e bem sucedido, no que diz respeito ao acompanhamento e controle do estado nutricional a fim de evitar que o processo de envelhecimento seja visto como algo maléfico ou o fim da vida e dos prazeres de viver, se tratando de saúde ou da ausência dela.

Segundo Ferreira et al., (2012) e Assis (2002), a promoção do envelhecimento saudável se mostra complexa, pois gira em torno não apenas da qualidade de vida, mas também do acesso a serviços de saúde que auxiliem o idoso neste processo. Sendo assim, a educação nutricional é uma das principais ferramentas no que diz respeito à promoção da saúde, modificando hábitos alimentares e dando autonomia para que os indivíduos possam fazer escolhas saudáveis. (Criscuolo, 2009).

São muitos os fatores identificados que contribuem para a elevação dos níveis pressóricos, entre os quais se destacam idade avançada, obesidade, consumo excessivo de álcool, sedentarismo, dislipidemias, diabetes mellitus e alto teor de sódio na alimentação (Martins \& Fé, 2020).

O controle da hipertensão por meio da Educação Alimentar e Nutricional, pode ser trabalhado e feito por meio de práticas como: controle de peso corporal, de uma dieta adequada com baixo teor de sódio, diminuição do consumo de alimentos ultra processados e aumento da ingestão de frutas e verduras.

Cabe ao profissional de saúde orientar mudanças na dieta habitual visando à adequação do consumo energético, dos macro e micronutrientes, principalmente de potássio $(\mathrm{K})$, cálcio $(\mathrm{Ca})$ e magnésio $(\mathrm{Mg})$ associado à redução do consumo de sódio $(\mathrm{Na})$, das gorduras saturada e trans, além do incremento do consumo das gorduras mono e poli-insaturadas e das fibras. (Brasil, 2013).

A relevância da EAN para o fortalecimento das ações de nutrição, auxiliam no empoderamento dos indivíduos e no desenvolvimento da autonomia diante de suas escolhas alimentares (França \& Carvalho, 2017).

Assim, essas informações ressaltam a importância da educação nutricional atrelada a uma vigilância alimentar, com o intuito de evitar o aumento dos riscos relacionados a presença e desenvolvimento de doenças crônicas (Miranda et al., 2017).

É importante relatar que a Nutrição exerce um papel fundamental no cotidiano das populações, presente desde a 
Research, Society and Development, v. 10, n. 9, e8010917832, 2021

(CC BY 4.0) | ISSN 2525-3409 | DOI: http://dx.doi.org/10.33448/rsd-v10i9.17832

formação de um ser, nos processos de saúde-doença e até o fim da vida com o intuito principal de promover saúde, prevenir agravos de doença e atuar na manutenção dos órgãos vitais de uma maneira geral.

Portanto, pode-se dizer que a Nutrição está diretamente relacionada a qualidade de vida sendo ela para pessoas sadias ou enfermas, atuando em todas as fases da vida salientando então o papel do profissional nutricionista na Nutrição Humana, sendo algumas de suas responsabilidades promover saúde através do planejamento individual e de coletividades, das refeições diárias, da dietoterapia de acordo com a patologia, elaboração de cardápios, controle da ingestão alimentar, manutenção e recuperação do estado nutricional, compreendendo as interações bioquímicas e fisiológicas dos nutrientes no corpo humano, além de atuar diretamente no tratamento e controle de agravos das doenças crônicas não transmissíveis que acometem desde as crianças, adolescentes, jovens, gestantes até os idosos que necessitam de dietas específicas para a sua condição.

\section{Conclusão}

Conclui-se que a Educação Alimentar e Nutricional, poderá atuar de forma ativa no processo de envelhecimento humano, e pode ser considerada uma estratégia para promover uma alimentação adequada e saudável, e dessa forma promover saúde e qualidade de vida, trabalhando com ferramentas interdisciplinares e ativas voltadas a pessoa idosa.

Propondo-se a abordar temas que façam com que os idosos desenvolvam autonomia e responsabilidade diante das suas escolhas alimentares e melhorando sua relação com a comida, modificando seus hábitos através de pequenas alterações no seu dia a dia, introduzindo mais frutas, verduras, legumes, cereais integrais, alimentos com baixo teor de sódio, evitando alimentos ultraprocessados, ricos em sódio e menos prejudiciais à saúde.

Portanto, trabalhar a Educação Alimentar e Nutricional com idosos hipertensos pode ser enriquecedora e proveitosa, visto que o seu principal objetivo é dar autonomia aos seus educandos. E uma das possibilidades de continuação é a realização de outras pesquisas sobre o assunto, visando trabalhar vantagens e fragilidades que a EAN apresenta, seja na elaboração de conceitos ou de conhecimentos repassados ao longo do tempo.

\section{Referências}

Alencar, A. P. A. et al (2016). Impacto do programa mais médicos na atenção básica de um município do sertão central nordestino. Revista Eletrônica Gestão \& Sociedade, 10, 1290-1301

Alencar, M. S. S., Barros, J. F. O. \& Carvalho, C. M. R. G. (2008). Os aportes sócio-políticos da educação nutricional na perspectiva de um envelhecimento saudável. Revista de Nutrição, 21, 369-381

Assis, Mônica de (Org.). et al. (2002). Promoção da saúde e envelhecimento: orientações para o desenvolvimento de ações educativas com idosos. CRDE UnATI UERJ,.

Boog, M. C. F. (2013). Educação em Nutrição: integrando experiências. Komedi.

Brasil. (2012a). Ministério da Saúde. Secretaria de Atenção à Saúde. Departamento de Atenção Básica. Política Nacional de Atenção Básica. Ministério da Saúde,

Brasil. (2012b). Ministério do Desenvolvimento Social e Combate à Fome. Secretaria Nacional de Segurança Alimentar e Nutricional. Marco de Referência de Educação Alimentar e Nutricional para as Políticas Públicas. Ministério da Saúde.

Brasil. (2013). Ministério da Saúde. Secretaria de Atenção à Saúde. Departamento de Atenção Básica. Política Nacional de Alimentação e Nutrição (PNAN). Ministério da Saúde.

Brasil. (2013). Ministério da Saúde. Secretaria de Atenção à Saúde. Departamento de Atenção Básica. Estratégias para o cuidado da pessoa com doença crônica: hipertensão arterial sistêmica/ Ministério da Saúde, Secretaria de Atenção à Saúde, Departamento de Atenção Básica. - Brasília: Ministério da Saúde,.128 p.: il. (Cadernos de Atenção Básica, n. 37).

Cervato, Ana Maria et al. (2005). Educação nutricional para adultos e idosos: uma experiência positiva em Universidade Aberta para a Terceira Idade. Revista de Nutrição, 18, 41-52

Corbett, Mark S., Higgins, Julian P.T. \& Woolacott, Nerys F. (2014). Assessing baseline imbalance in randomised trials: implications for the Cochrane risk of bias tool. Research Synthesis Methods, 5, 79-85. 
Research, Society and Development, v. 10, n. 9, e8010917832, 2021

(CC BY 4.0) | ISSN 2525-3409 | DOI: http://dx.doi.org/10.33448/rsd-v10i9.17832

Criscuolo, Carolina. (2009). Contribuições da educação nutricional junto a um grupo de idosos. 199 f. Tese (Mestrado em Alimentos e Nutrição) - Faculdade de Ciências Farmacêuticas, Universidade Estadual Paulista “Júlio de Mesquita Filho", Araraquara.

Duarte, L. T. (2008). Envelhecimento: processo biopsicossocial. Trabalho de Conclusão de Curso Virtual (“Educación para el Envejecimento") - TIEMPO (El portal de Lapsico gerontoilogía).

Ferreira, Lucas L. et al. (2012). Perfil sociodemográfico e funcional de idosos institucionalizados. Estudos Interdisciplinares sobre o Envelhecimento, 17, 373386.

Ferreira, O. G. L., Maciel, S. C., Costa, S. M. G., Silva, A. O. \& Moreira, M. A. S. P. (2012). Envelhecimento Ativo E Sua Relação Com A Independência Funcional. Texto Contexto Enferm, 21, 513-8

França, C. J. \& Carvalho, V. C. H. S. (2017). Estratégias de educação alimentar e nutricional na Atenção Primária à Saúde: uma revisão de literatura. Saúde Debate, 41, 932-948

Greenwood, S. A. \& Fonseca, A. B. (2016). Espaços e caminhos da educação alimentar e nutricional no livro didático. Ciência e Educação. 22, 201-218.

Hardt, Ana Carolina et al. (2006). Um olhar a terceira idade: promovendo nutrição e saúde no asilo Irmão Joaquim. In: Encontro Nacional Dos Grupos Pet, 11., 2006, Florianópolis. Anais. Editora UFSC.

Henriques, K. C. A., Figueiredo, P., Goyatá, S. L. T., Terra, F. S. \& Loureço, D. S. (2015). Plano de ação alimentar e nutricional da pessoa idosa. Revista Baiana de Enfermagem, 29, 50-58

Higgins, Julian P. T. et al. (2011). The Cochrane Collaboration's tool for assessing risk of bias in randomised trials. Bmj, 343 , d5928.

Jaime, P. C. et al. (2011). Ações de alimentação e nutrição na atenção básica: a experiência de organização no Governo Brasileiro. Revista de Nutrição, 24, $809-824$

Lima, A. M. P., Ramos, J. L. S., Bezerra, I. M. P., Rocha, R. P. B., Batista, H. M. T. \& Pinheiro, W. R. (2016). Depressão em idosos: uma revisão sistemática da literatura. $R$ Epidemiol Control Infec, 6, 97-103.

Lima, F. K. S. M. \& Pietsak, E. F. (2016). Saúde do idoso: atividade física, alimentação e qualidade de vida. Revista Extendere, 4, 49-62

Lopes, Livia Amaral Alonso. (2016). Risco de viés em ensaios controlados randomizados de intervenções farmacêuticas.

Martins, A. B. et al. (2014). Atenção Primária à Saúde voltada as necessidades dos idosos: da teoria à prática. Ciência \& Saúde Coletiva, 19, 3403-3416

Martins, J. F. M. S. \& Fé, M. A. M. (2020). Intervenção nutricional em idosos hipertensos pertencentes à unidade básica de saúde lagoa. Paulistana (PI).

Miranda, R. N. A., Carvalho, E. P., Amorim, Y. R., Santos, K. S. \& Serrão, F. O. (2017). Conhecendo a saúde Nutricional de idosos Atendidos em uma Organização não Governamental, Benevides / PA. Revista Conexão UEPG, 13, 512-29

Najas, M. S. \& Nebuloni, C. C. (2005). Avaliação Nutricional. In: Ramos LR, Toniolo NJ. Geriatria e Geontologia. Manole

Nascente, F. M. N., Jardim, P. C. B. V., Peixoto, M. R. G., Monego, E. T., Moreira, H. G., Vitorino, P. V. O., Souza, W. K. S. B. \& Scala, L. N. (2010). Hipertensão arterial e sua correlação com alguns fatores de risco em cidade brasileira de pequeno porte. Arquivos Brasileiros de Cardiológicos. 95 Epub.

Oliveira, M. A. C. \& Pereira, I. C. (2013). Atributos essenciais da Atenção Primária e a Estratégia Saúde da Família. Revisa Brasileira de Enfermagem, 66, $158-164$

Santos, H. A., Gomes, S. C. S. \& Lima, R. J. C. P. (2018). Educação em saúde: uma estratégia no cuidado com idosos hipertensos. Pesquisa em Foco, 23, 194206

Santos, R. D., Gagliardi, A. C. M., Magnoni, X. H. T. \& Magnoni, C. D. et al. (2013). I Diretriz sobre o consumo de gorduras e saúde cardiovascular. Arq. Bras. Cardiol. 100

Sociedade Brasileira De Cardiologia - SBC. (2016). VII Diretriz Brasileira de Hipertensão Arterial. Arq. Bras. de Cardiol, 7, 1-103.

Shea, Beverley J. et al. (2017). AMSTAR 2: a critical appraisal tool for systematic reviews that include randomised or non-randomised studies of healthcare interventions, or both. $b m j, 358, \mathrm{j} 4008$.

Shea, Beverley J. et al. (2009). AMSTAR é uma ferramenta de medição confiável e válida para avaliar a qualidade metodológica de revisões sistemáticas. Jornal de epidemiologia clínica, 62, 1013-1020.

Souza, P. C. D., Amorim, G. V. Q., Lima, A. A. F., Temoteo, M. A. S. \& Araújo, I. L. S. B. (2018). Impacto de ações de educação alimentar e nutricional no perfil antropométrico e consumo alimentar de hipertensos. Revista eletrônica Estácio Recife, 4, 1-11.

White, H. J. \& León, L. M. (2014). Orientações nutricionais em serviços de saúde: a percepção de idosos portadores de hipertensão e diabetes. Demetra, 9 , 867-880. 